\title{
Investigating the Affordances of Wellness mHealth Apps
}

\author{
Moayad Alshawmar \\ Worcester Polytechnic Institute \\ malshawmar@wpi.edu
}

\author{
Haadi Mombini \\ Worcester Polytechnic Institute \\ hmombini@wpi.edu \\ Isaac Vaghefi \\ Pace University \\ sashrafvaghefi@pace.edu
}

\author{
Bengisu Tulu \\ Worcester Polytechnic Institute \\ bengisu@wpi.edu
}

\begin{abstract}
Previous research on the effectiveness of wellness mHealth apps focused on the design and features of such apps and paid insufficient attention to how the whole relationship between the apps and users impact use. Using affordance theory, we investigated what wellness mHealth apps afford to users and why these affordances are not actualized by all users. We conducted a qualitative study, collecting data from apps' reviews and from fifteen participants who used multiple wellness mHealth apps. Our grounded theory analysis revealed four shared affordances (promoting goals, comparing oneself to others, coaching, and nurturing) related to the use of wellness mHealth apps and three immediate concrete outcomes (habit formation, self-awareness, and goal attainment) reached after the affordances were actualized. Nonetheless, factors such as information overload, aesthetic appreciation, and users' characteristics may impact users' actualizations of the shared affordances and prevent some users from reaching their immediate concrete outcomes.
\end{abstract}

\section{Introduction}

Mobile health apps are becoming increasingly popular, and the evidence for their impact on individuals' health, wellness, and nutrition is accumulating [1]. Such apps allow access to health information [2], support people in their recovery from chronic diseases [3] and provide them with access to healthcare at a lower cost [4]. Using these apps, people can, for instance, follow plans for workouts and track their diet and nutrition [5]. It is also shown that, while wellness mHealth apps can improve people's general well-being, they cannot intervene to manage their conditions or diseases [6]. These apps have the potential to improve people's wellbeing by allowing for more efficient data collection related to their activities and health-related habits such as walking, sleeping, and drinking liquids $[7,8]$.

Despite the various benefits of mHealth apps, existing review studies have reported mixed results in terms of these apps' effectiveness for users [9-15]. For example, one study showed that less than $30 \%$ of users who used a weight loss app for a continued period may see improvements in their weight, body fat, and BMI [16].

This issue has created an opportunity for researchers to investigate the use of mHealth apps and to explore ways to improve them $[17,18]$. The existing studies have focused on difficulty of use [9], design features or functions [8], and the impact of these apps on users' health [3]. However, such studies have not paid close attention to how the users, in light of their particular characteristics and goals, interact with these apps.

Affordance theory focuses on what an object delivers to the user rather than on the object's predefined features [19]. In the context of technology use, it has been argued that, to understand user behaviors, the features of the technology and those of the users should be studied based on the relationship a user have with an app's features [20]. This means a user or a group of users, based on their intentions, perceptions or goals, may use a technology in a way that may or may not be aligned with the developers' intended purpose of the technology [21-24]. In this way, affordance theory facilitates understanding the relationship between users and an information technology (IT) artifact, providing a useful lens to discover how such relationships can impact users' interactions with wellness mHealth apps.

Identifying the affordances of wellness mHealth apps would reveal how users' interaction with the apps could lead to accomplishing the ultimate purpose of the apps, which is to improve users' well-being. In this study, we aimed to answer two questions: 1) what are the wellness mHealth apps' shared affordances (i.e. 
the same affordance being actualized by many users using the apps) and the associated outcomes that are reached after these affordances are actualized? 2) What are the factors that impact (facilitate or inhibit) users' actualizations of the shared affordances?

In order to respond to these questions, we drew upon the affordance theory [19] and the concept of shared affordances (when a group of users actualize similar affordances while interacting with the IT artifact) [25]. Despite the increasing attention paid to technology affordances in IS research, studies focusing on wellness mHealth apps and their affordances have been limited. Better understanding of these affordance is critical because it will help clarify how these apps can be useful to a larger population of users.

\section{Theoretical background}

\subsection{Mobile health and wellness apps}

The accessibility of smartphones has made possible the wider use of many health-related apps and the sharing of such apps with a great number of people who seek to improve their health [26]. Some of these apps are designed to manage chronic diseases or to treat a patient [3], while others are developed to promote healthy behaviors to improve users' wellness [27]. Wellness mHealth apps help to enhance users' well-being by encouraging and tracking physical activity [27], weight management [28], dietary interventions [30], stress management [30], and more. Wellness mHealth apps may vary in their features and designs, but they share one ultimate goal, which is to improve users' well-being. Currently, developers of mHealth apps do not always have the clinical domain expertise [31]. Furthermore, users engage with the wellness app features immediately, with no prior knowledge of the expertise of the developer who hopes to lead them to their health goals [31]. Hence, it is crucial to understand how these apps help to improve people's well-being. Our goal is to investigate the relationship between the app and the individual, and to study the apps' affordances and how they can facilitate or prevent users from accomplishing their ultimate health goals.

\subsection{Affordance theory and IT}

Affordance theory, as proposed by Gibson [19], argues that animals (including humans) interact with an object in consideration of their relation with the object in its given environment and what the object can provide for them, based on their perception. In line with this view, Leonardi [32] argued that "people do not interact with an object prior to or without perceiving what the object is good for." According to the theory, the way an individual views certain objects will subsequently determine the importance of the objects to meet the individual's needs, above and beyond what the objects' features are. In this way, the theory highlights the important distinction between features (the functions of technology) and affordances (the potential for achieving a goal by using the technology's functionalities). Although affordances are typically studied at the individual level of analysis, they can also emerge a higher level of analysis (e.g. teams, groups etc.) [24]. Shared affordance, by definition refers to the similar ways a group of individuals may interact with a technology (i.e. shared relationships between user and artifact) [25].

Strong and Volkoff [33] advanced Gibson's affordance theory using a process-based definition of affordance as "the potential for behaviors associated with achieving an immediate concrete outcome and arising from the relation between an object and a goaloriented actor or actors." This definition splits the process of an affordance into "potential for action," "the action itself" (actualizing affordances), and the "immediate concrete outcome" (the state or condition reached immediately after the affordance/s are actualized) (i.e. potential $\rightarrow$ actualization $\rightarrow$ immediate concrete outcome). According to this view, in the first step of the process, a user or a group or users must have the potential to use an IT artifact [20]. To transform the user's potential into an affordance, the user must carry out an action using the IT-artifact (e.g. accessing, observing, and/or monitoring data) [20]. Once the action or actions are completed, a user will reach an immediate concrete outcome [20]. Without a user's action (actualization), the immediate concrete outcome will not occur for that particular user or a group of users [20].

Previous research has investigated design-related factors that could help users accomplish their ultimate wellness goals using mHelath apps [9-15]. Yet, how users utilize these apps to achieve their wellness goals is still unclear. To fill this gap, in this study, we explore the affordances of wellness mHealth apps and their immediate concrete outcomes. While uncovering the apps' shared affordances, we aim to identify factors that impact users' actualizations of the shared affordances, following Volkoff and Strong [20] guidelines. We will apply these theoretical foundations, specifically the concept of shared affordance that takes into account what technology affords to a group of users at the same time, while using multiple wellness mHealth apps. In this way, 
affordance theory can help illustrate the technology's usefulness to a user or a group of users [20].

Understanding the shared affordances of wellness mHealth apps and their immediate concrete outcomes can ultimately pave the way to describe how such technologies can be useful for improving users' wellbeing. Furthermore, it is critical to provide an overview of how users experience the actualized affordances and why some users do not actualize the shared affordances. Accordingly, we will investigate the factors that impact users' actualizations of the shared affordances.

\subsection{Affordance theory in IS research}

Affordance theory has been used in various research studies in the IS domain [34-36] and in contexts such as software development [37, 38], human-computer interaction [39], and social media [40, 41]. Regarding the mHealth domain, studies have used affordance theory to explain phenomena related to mHealth apps with a focus on particular settings [42], specific chronic conditions [43], particular diseases [44], and overall wellness [26].

In the wellness domain, a few studies have used affordance theory. For example, one study used goal content theory and affordance theory to uncover the impact of users' goals for exercising (such as enjoyment, competence, and body appearance) on the preference for using certain fitness application features [45]. The findings of this study revealed that users who use these apps for enjoyment and competence are more likely to use features that allow them to see their exercise progress and to make social connections with others [45], while those who use the app to change their body appearance are not interested in the socializing features [45]. In their study of the impact of a gamified mHealth app on users' motivation and behaviors to exercise, Wong and Kwok [46] applied both affordance and selfdetermination theory. They hypothesized that competence affordance coming from "leaderboard/achievement badge" features, as well as relatedness affordance coming from "like function/from a team" features have a positive relationship with exercise motivation.

Prior research has shown the usefulness of the affordances theory for explaining how users interact with the mHealth apps to reach their goals [46-48]. However, the related studies are limited in their investigation as they typically focus on a single application (exercise or diet) or take into account a limited number of goals and features. We argue that, while they provide valuable insights, the findings of such research cannot be generalized without studying affordances across multiple apps. Hence, we apply affordance theory [49] to explore the shared affordances of multiple wellness mHealth apps.

\section{Methodology}

To investigate the shared affordances of wellness mHealth apps, their concrete immediate outcomes, and factors impacting users' actualizations of the shared affordances, we followed a qualitative methodology. Our study included two rounds of data collection. In the first round, we recruited participants to use a wellness app of their choice and interviewed them before and after 14 days of use. In the second round, we collected and analyzed reviews from Apple's App Store for the apps used in the first phase of the study. We followed a grounded theory approach [50] to analyze these data.

\subsection{Study sample and data collection procedures}

Phase 1 - Interviews: We recruited participants via an open call in two large US universities. To be selected, the participants were required to have no significant chronic diseases. This helped us ensure that the motivation of a participant would not be significantly different from that of a typical, healthy mHealth user. Participants were screened over the phone or face-to-face for the study. The study was approved by the Institutional Review Board (IRB) at both universities.

We conducted semi-structured interviews (pre and post) to capture users' perceptions and behaviors in using wellness mHealth apps after the 14-day use period. At t1, the participants were asked to select a wellness Health app and a specific health goal that could be reached using mHealth tools. At $\mathrm{t} 2$, the participants were asked to (1) describe their experience with the apps, (2) reflect on their goals and motivation, (3) assess whether the application had helped them achieve their goals, and (4) make any design or improvement suggestions for application designers. The interviews took, on average, 21 minutes (13-47 minutes) and were audio recorded. The participants received $\$ 50$ gift cards as an incentive at the end of the study period. Table 1 presents the apps included in this phase of the study.

Phase 2 - Review comments in the App Store: In order to enhance our understanding of the affordances and complement our primary interview data, we supplemented our primary data with review comments posted on Apple's App Store. Looking at the apps used by participants in phase 1 , we recorded every comment 
posted in a one-year period, from September 2018 to September 2019. A total of 14601 comments made by 14573 unique users were recorded, using a Python script from Shazam (A GitHub repository). We considered App Store data from 7 countries (United States, France, Austria, United Kingdom, Italy, Russia and Germany). We sorted the comments by country, app title, user rating, and App version; we translated (using the same script) the comments that were not in English. After removing reviews with one-star ratings (i.e. very low ratings) to avoid biases, we were left with 6237 review comments (4669 from US, 1470 1534 from England, and the rest from the other countries) written by 6213 unique users. Table 1 presents the percentage of comments posted for each app.

Table 1. Study Participants and Selected Apps.

\begin{tabular}{|c|c|c|c|}
\hline ID & mHealth App & Category & $\begin{array}{c}\text { App store } \\
\text { Comments }\end{array}$ \\
\hline S1 & $\begin{array}{c}\text { Samsung } \\
\text { Health }\end{array}$ & Activity & $2 \%$ \\
\hline S2 & ASICS & Activity & $.2 \%$ \\
\hline S3 & Habit Ball & Habit & $2 \%$ \\
\hline S4 & Aura & Mindfulness & $5 \%$ \\
\hline S5 & Garmin & Activity & $8 \%$ \\
\hline S5 & PlantNanny & Hydration & $4 \%$ \\
\hline S6 & Fitbit & Activity & $7 \%$ \\
\hline S7 & $\begin{array}{c}5-\text { minute home } \\
\text { workout }\end{array}$ & Activity & $2 \%$ \\
\hline S8 & Headspace & Mindfulness & $8 \%$ \\
\hline S9 & Relax (lite) & Mindfulness & $.1 \%$ \\
\hline S10 & $\begin{array}{c}\text { Weight } \\
\text { Watchers }\end{array}$ & Diet & $9 \%$ \\
\hline S11 & TnS Lite & Sleep & $.2 \%$ \\
\hline S12 & Map My Walk & Activity & $5 \%$ \\
\hline S13 & ARise & $\begin{array}{c}\text { Diet/physical } \\
\text { activity }\end{array}$ & $1 \%$ \\
\hline S14 & My net diary & Diet & $14 \%$ \\
\hline S15 & $\begin{array}{c}\text { Nike + } \\
\text { Training }\end{array}$ & $\begin{array}{c}\text { Physical } \\
\text { activity }\end{array}$ & $31 \%$ \\
\hline
\end{tabular}

\subsection{Data Analysis}

Interviews: Interviews were transcribed and recorded in NVivo for analysis. Participants were assigned a unique identifier, and all identifiable information was removed from the transcripts before the analysis. We took a grounded theory approach and coded these data in an iterative fashion [50]. First, we completed an open coding phase, which created 35 codes describing users' perceptions, evaluations, and expectations for the apps. We compared the codes and created 14 new codes, resulting in a total of 49 opencoded concepts. During the axial coding phase, we analyzed each code in detail and mapped any phrase, sentence, or paragraph that we found relevant to affordances to these codes. Each code was scrutinized based on the concept of affordances, merged with other equivalent codes, and then discussed among authors, until we reached a consensus. Specifically, our analysis focused on participants' comments about what the apps had afforded or could afford them, what the immediate concrete outcomes reached after actualizing the affordances were, and what could impact some users' potentials of actualizing the shared affordances. For the codes that we identified as affordances, immediate concrete outcomes, and factors impacting some users' potentials, we searched for appropriate terminologies reported in the literature.

App reviews: After analyzing our interview data, we started analyzing the 6237 review comments from 6213 unique users. Following the same approach (grounded theory), the first author read through all the 6237 review comments and coded them using the existing codes while observing for new emerging themes (see key themes in Figure 1). The first author associated each comment with the relevant affordances and their outcomes. Users' evaluations of the shared affordances were captured as factors that stopped them from actualizing or encouraged them to actualize these affordances. Some comments provided evidence of multiple affordances. The identified affordances, their immediate concrete outcomes, and the factors that impacted users' actualizations were compared with those identified during Phase 1, and similar ones were combined together after all authors had discussed and confirmed the similarities.

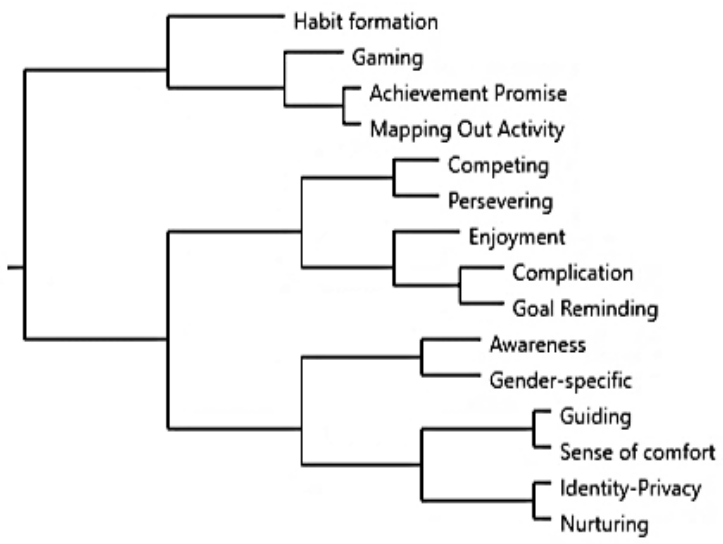

Figure. 1 Key themes identified based on the qualitative analysis.

\section{Results}

Based on the analysis of our primary and secondary data, we identified four shared affordances related to the use of wellness mHealth apps, namely 
promoting a goal, comparing self to others, coaching, and nurturing. In addition, our results pointed to three immediate concrete outcomes - habit formation, selfawareness, and goal attainment - that can be reached after actualizing the shared affordances (see Vignette 1.). Finally, our analysis revealed three factors that influence users' actualizations of the shared affordances and the reaching of their outcomes, namely information overload, aesthetic appreciation, and users' characteristics. Most participants actualized these affordances, that is, they used the app and experienced the intended action potentials. In contrast, few participants expressed their limited (or no actualization of affordances from use) although they initially were aware of the app's shared affordances. For these users, their experience appeared to be impacted by factors related to the design of the apps or the users' characteristics of preference and needs. We provide details about these results next.

\subsection{Affordances of wellness mHealth apps}

Promoting a goal (afforded to 7 participants and 14 app reviewers): We found that promoting a goal is an affordance initiated through users' interaction with the apps' reminding features [51]. When participants and the apps' reviewers received reminder messages as texts or colored light, a sense of encouragement to carry out the task was afforded. Our findings showed a preference for certain types of messages that contained positive language over others that made users feel that a task or action was necessary.

"This has little red lights that kind of pop up. So that kind offorced me, hey I haven't walked for a while or maybe I'll do a walk around the building. "/ S5.

"I think positive words b/c like it would give me notifications, but it would say "you missed daily lunch". I think a more positive language like "what did you have for lunch?" would make me want to instead of it being homework."/S13.

Comparing self to others (afforded to 6 participants and 12 app reviewers): This affordance relates to how individuals compare their performance to that of others [52]. Our analysis showed that some wellness mHealth apps provide users with features that enable them to see how other users are progressing on similar goal-oriented tasks (e.g., walking longer distances). Although the majority of our participants were interested in viewing the progress of their friends, relatives, or strangers who were using the same application, some users found such a comparison and self-evaluation irritating (also refer to the factors influencing users' potentials actualizing shared affordances).

"I like it. And those other pieces are interesting too because you can benchmark yourself against other users on the Samsung app. It shows you based on your age, your gender everything"/ S1.

"The interaction from others on connect to motivate you and seeing your weight there going down. Love it."'/ App reviewer

Coaching (afforded to 7 participants and 46 app reviewers): Some wellness mHealth apps provided users with detailed information on how to complete certain tasks that would help them achieve their goals. This information was provided in the form of tutorials or visual instructions such as on-screen guides. This information is coaching users to accomplish the required task. Users who actualized this affordance reported such coaching helpful, because it prevented unintended negative consequences (e.g., injury in activity tracking apps).

"It gives you guidelines in terms of how your body should be positioned to do certain things. If you don't know the right position you could be doing more damage than good." 59 .

"I just turned, and I really needed someone to coach me and to go through this with me. I know she is not even here, but I could feel her there with me."' App reviewer

Nurturing (afforded to 1 participant and 20 app reviewers: Nurturing affordance is when users are taking care of an app's avatar or fictional character. [53]. This affordance is shown to encourage feelings of friendship, identification, responsibility, and heightened sense of control over the fictional character's actions [54]. In our sample, some of the apps (e.g. PlantNanny) afforded users a kind of responsibility. For instance, while using PlantNanny users work toward growing a virtual plant upon accomplishing the required task, which is drinking water. By drinking the required amount of water, the virtual plant will grow vs. die out of dehydration. This kind of responsibility for saving the plant initiated an empathetic feeling that motivated users to drink water.

"probably if I ever just had a couple of busy days and the plant died, I think that would traumatize me." /S9.

"Plant nanny has helped me drink as much water as I need every day by giving me a cute plant to look after and take care and help take care of myself "/ App reviewer 


\subsection{Immediate concrete outcomes of actualized affordances}

Habit Formation (reached by 4 participants and 12 app reviewers): Habit formation is an outcome observed when an actor practices a new behavior in a chosen context, and the habit develops as the actor learns through repeating that behavior and strengthening the context-behavior association [55]. As illustrated in the two examples below, our study showed how the affordances of wellness mHealth apps shaped users' daily activities. These daily activities became habits in users' lives that helped them to accomplish their goals for using the apps.

"... I found myself doing things like parking further away, walking, taking extra steps...."/ S12.

"Been using the app for months now lost a stone in a month and maintained ever since. We use it every day for tracking and recipes. The $W W$ recipes are fantastic and have totally changed my whole family's approach to food."' App reviewer

Self- awareness (reached by 11 participants and 46 app reviewers): Self-awareness means that individuals can sense their behaviors and are aware of their actions, abilities, and traits, and they consciously control them [56]. In our study, certain affordances of wellness mHealth apps, such as comparing self to others, enabled users to see clearly how they were functioning. We found users became aware of their health-related behaviors and their approach toward achieving them. According to the self-determination theory, self-awareness is critical is approaching goals and is positively associated with one's ability to function effectively [56]; accordingly, we found that people prefer to receive more information of their health-related behaviors via use of these apps.

"I like it. And those other pieces are interesting too because you can benchmark yourself against other users on the Samsung app. It shows you based on your age, your gender, everything."/S1

"I highly recommend WW to anyone who has challenges with their body and the food they eat. I have learned a lot about what makes a difference and what hurts me in calories and nutrition and life."/ App reviewer

Goal Attainment (reached by 9 participants and 32 app reviewers): Our participants described how the reminding, guiding, and nurturing affordances of wellness mHealth apps enabled users to see their progress against their goals, to see how close they were to attaining their goals. A similar affordance was uncovered in a study of affordances to break smoking habits [43] in which authors found that visibility of effort to quit smoking on a progress page in the form of graphical representations motivated users to continue working toward their goal.

"You get like notifications throughout the day. It might seem like you're halfway to goal or 15 minutes left to goal. At the end of the day at some point you will say oh you reach your goal right."/ S1.

"It is a really interesting game. It doesn't ask you for much information about yourself like other apps may do. It gives you day by day guides so you can work up to the highest level of working out."/ App reviewer

"I want to be able to track my goals on my phone app. It is not always convenient to go to the website, and Garmin US website isn't very mobile friendly either. "/ App reviewer

Comparing self to others (AFF)

"I like it. And those other pieces are interesting too because you can benchmark yourself against other users on the Samsung app. It shows you based on your age, your gender everything"/ S1

Self-awareness (outcome)

Nurturing (AFF)

"Really helpful and because I have a huge love for taking care of plants it really has me actually taking care of myself. Super happy with it!!."'/ App reviewer

Coaching (AFF)

Goal Attainment (outcome)

"Been using the app for months now lost a stone in a month and maintained ever since. We use every day for tracking and recipes. The $W W$ recipes are fantastic and have totally changed my whole family's approach to food" App Reviewer

Habit Formation (outcome)

Vignette 1. Affordances of wellness mHealth apps and their immediate concrete outcomes.

\subsection{Factors impacting mHealth wellness apps' shared affordance for some users}

Information Overload: "Information overload occurs when information received becomes a hindrance rather than a help, even though the information is potentially useful" [57]. In the context of mHealth applications, presenting the information is regarded as an important consideration for developers [58]. Improving the presentation can reduce information overload and thus improve the quality of 
users' decision making [59]. We found that when participants were experiencing information overload, they looked for the option to customize their applications to avoid information overload. The participants appreciated applications' providing the option to customize the interface. Most of these customizations were focused on simplifying the interface to avoid information overload.

"My whole dashboard is challenge information and requests that I donut care to see. The information I actually care about is too small and at the bottom of the screen."./ App reviewer

"For the T\&S app, I think the customizable side of it and being able to track exactly what I wanted is probably its biggest feature and something that I've been missing."/ S11.

"This update is beyond dreadful. A dashboard should show keys stats at a glance... I shouldn't have to scroll to see most of the information! Pretty basic."' App reviewer

Aesthetic appreciation: Aesthetic appreciation refers to "an interplay between an individual's visual, auditory, olfactory, tactile, haptic, and even proprioceptive systems" [60]. Aesthetic appreciation can be seen through the visual appearance of the components and features of the product. Like other products, mHealth applications may be evaluated not only for their usability but also for their aesthetic look and feel. It has been shown that the look and feel of the apps (e.g., I liked the way the dashboard looked) are as important as their use and application. Our findings suggest that although it is important to have a well-designed user-friendly application that functions smoothly, a good sense of design also seemed to be appreciated by our participants and the apps' reviewers in wellness mHealth applications. At the same time, unfavored aesthetics in apps have impacted participants' actualization of their affordances.

"I liked the way the dashboard looked, it was just so clean and so I said alright well I'll download that. Give it a try. And I've been really happy with that."/S6

"I do not like the new dashboard and would prefer the option to revert back to the old one, which was more aesthetically pleasing!! Please add in an option to revert Fitbit!'”/ App reviewer

Users' characteristics: Different types of users have different preferences of things they like and dislike. In the context of wellness mHealth apps' affordances, we found that some of the affordances essentially do not match with users' characteristics. In other words, the user does not consider the shared affordances to be useful to him/her. Users' preferences for certain affordances over others may impede them from actualizing the shared affordances.

"Social media aspects like you can find friends and see who's walked more. Yeah but that's dumb. I think it helps some people but not for me." (S2, comparing self to others AFF)

"... it actually had a feature that you could set reminders. But I don't really use anything on my phone like that ... like it would drive me nuts. OK so I don't have any reminder." (S8, promoting goal, AFF)

"I like making my own schedule and putting in my own exercises. Cause if there is 1 that works well, I know to put that in and if there is one that does not work, I can take it out, but you can't really do that if there is a set program for you." (S2, coaching)

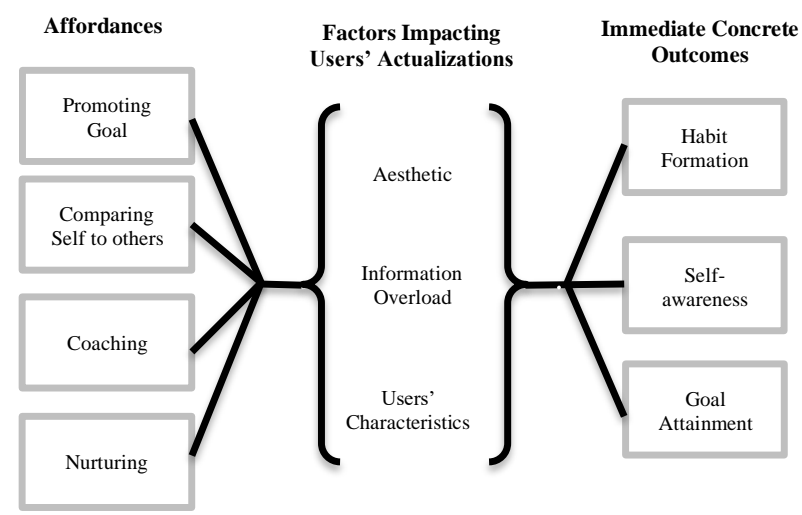

Figure. 2 Affordances of Wellness mHealth, factors, and immediate concrete outcomes.

\section{Discussion}

Prior studies have indicated that although mHealth apps show great promise in improving the wellness of some users, they can be ineffective for most users [9-15]. For instance, these apps can be abandoned, misused avoided, or even resisted after the initial adoption [61]. Few prior studies have investigated this phenomenon using affordance theory [46-48]. These studies have focused on either a specific app or one particular condition (e.g., disease or chronic illness) to uncover some of the wellness mHealth affordances [46-48]. Other studies have focused solely on features underlying the affordances of the apps [39].

Following Volkoff and Strong [20] guidelines for using affordance theory, we identified general affordances of multiple wellness mHealth apps and their immediate concrete outcomes (the state or condition reached immediately after the affordance/s are actualized) that were shared by a group of users. 
The findings add to the wellness mHealth app literature by 1) introducing shared affordances of multiple wellness mHealth apps, 2) identifying a set of immediate concrete outcomes that are reached after these affordances are actualized, and 3) illustrating that the immediate concrete outcomes may not be reached if users' actualizations are impacted by some factors. This is consistent with findings in the affordance literature that interaction with an IT artifact will enable a set of a affordances that are shared by number of users [25]. Our findings are consistent with the concepts that some wellness mHealth apps capabilities (e.g., enable users to see other progress) may afford nothing to some users [47]. We identified the shared affordances of wellness mHealth apps and explained that such affordances might be evaluated negatively or positively due to factors related to the design and to users' preferences.

We found that not all the identified affordances were afforded by all the included apps. For example, the nurturing affordance was afforded to few participants and the app reviewers. This affordance helped motivate users to start and to continue a task (e.g., to drink water regularly) until it became a habit. We argue that the nurturing affordance can be enabled by other apps in many ways that can motivate users to achieve their goals. Therefore, developers should consider enabling such useful affordances when they design wellness mHealth apps.

We also found that factors such as apps' aesthetics and information overload, along with users' characteristics, could facilitate or inhabit users' actualizations of the shared affordances. Our findings show that some affordances such as comparing self to others that do not fit with some users' characteristics. Other users do not like to see informative information and feel subject to overload when they see it. Hence, it is important to understand the users' preferences and what could inhibit or facilitate their actualizations.

Inhibiting users' actualizations of some affordances could impede users from reaching immediate concrete outcomes. Immediate concrete outcomes such as goal attainment, self-awareness, and habit formation could help motivate users to achieve the ultimate goal of using the apps (to improve wellbeing). Hence, it is important to ensure that wellness mHealth apps' affordances can be actualized in a way preferable to a user or to the majority of users. One way to enhance users' actualizations is to give them the option to customize some features (e.g. health information can be presented as informative content or as a visual figure) based on a user's preferences.

The affordance concept cannot be equated with the features of an app or the characteristics of users only. The affordances exist based on the relationship built between the users and the apps while users are using apps to improve their well-being. Hence, it is important for app designers to understand what is afforded to the majority of their users and how the affordances may not be as useful for all users. Also, it is necessary for designers to understand immediate concrete outcomes reached after users' actualizations and how they can be helpful for users to achieve the purpose of their apps.

\section{Limitation and future research}

In this study, our goal was to identify the affordances shared across participants and app reviewers using multiple wellness mHealth apps and to understand how the affordances lead users to achieve their goals. In our analysis, we excluded the affordances, immediate concrete outcome, and factors impacting actualization that were mentioned only marginally by participants and the apps' reviewers and instead focused on affordances that were salient. Nonetheless, these excluded affordances, immediate concrete outcomes, and other factors affecting affordance actualizations should be further explored in future research that includes larger populations and their interactions with a wider range of mHealth apps. Furthermore, linking the discovered affordances with the exact mHealth features that facilitate the affordance's appearance may help improve some wellness mHealth apps' design. We briefly explained how users' characteristics might impact their actualization of the shared affordances. Future research can study specific users' characteristics and examine why these users tend to actualize certain wellness mHealth apps affordances and not others. We illustrate that the identified affordances may, if actualized properly, lead users to improve their wellbeing. However, the time frame of two weeks in this study limited us to examine users' wellbeing improvement. Therefore, future research can extend the two weeks to measure users' wellbeing improvement when they actualize wellness mHealth apps affordances.

\section{Conclusion}

In this study, we aimed to contribute to the literature on wellness mHealth apps by identifying the shared affordances of apps which may impact users' achievement of the outcome of these apps. Drawing upon affordance theory and using data collected via two sources (interviews and reviews), we identified four affordances of wellness mHealth apps along with three immediate concrete outcomes that were reached 
after users actualized the affordances. Although our participants and reviewers of the apps shared similar affordances, some participants and app reviewers did not actualize the shared affordances as a result of three factors. The first is the users' characteristics that shape their preference for certain affordances as opposed to others. The second is the apps' design aesthetics. The third is the way the apps' presented information to users. Thus, we recommend researchers and developers to look into what their apps have afforded users and determine whether there are factors that impact users' actualization of the affordances.

\section{References}

[1] Marcolino, M.S., et al., The impact of mHealth interventions: systematic review of systematic reviews. JMIR mHealth and uHealth, 2018. 6(1): p. e23.

[2] Keutzer, L., S.G. Wicha, and U.S. Simonsson, Mobile Health Apps for Improvement of Tuberculosis Treatment: Descriptive Review. JMIR mHealth and uHealth, 2020. 8(4): p. e17246.

[3] Jongerius, C., et al., tested Mobile apps for breast cancer care: systematic review. JMIR mHealth and uHealth, 2019. 7(2): p. e10930.

[4] Wallis, L., et al., Integrating mHealth at point of care in low-and middle-income settings: the system perspective. Global health action, 2017. 10(sup3): p. 1327686.

[5] Milne-Ives, M., et al., Mobile apps for health behavior change in physical activity, diet, drug and alcohol use, and mental health: Systematic review. JMIR mHealth and uHealth, 2020. 8(3): p. e17046.

[6] Orji, R. and K. Moffatt, Persuasive technology for health and wellness: State-of-the-art and emerging trends. Health informatics journal, 2018. 24(1): p. 6691.

[7] Patel, M.S., D.A. Asch, and K.G. Volpp, Wearable devices as facilitators, not drivers, of health behavior change. Jama, 2015. 313(5): p. 459-460.

[8] Vaghefi, I. and B. Tulu, The continued use of mobile health apps: insights from a longitudinal study. JMIR mHealth and uHealth, 2019. 7(8): p. e12983.

[9] Aljuraiban, G.S., Use of Weight-Management Mobile Phone Apps in Saudi Arabia: A Web-Based Survey. JMIR mHealth and uHealth, 2019. 7(2): p. e12692.

[10] Guo, X., X. Zhang, and Y. Sun, The privacypersonalization paradox in mHealth services acceptance of different age groups. Electronic Commerce Research and Applications, 2016. 16: p. 5565.

[11] Hamine, S., et al., Impact of mHealth chronic disease management on treatment adherence and patient outcomes: a systematic review. Journal of medical Internet research, 2015. 17(2): p. e52.

[12] McKay, F.H., et al., Evaluating mobile phone applications for health behaviour change: A systematic review. Journal of Telemedicine and Telecare, 2016. 24(1): p. 22-30.
[13] Mohammadzadeh, N. and R. Safdari, Patient monitoring in mobile health: opportunities and challenges. Medical Archives, 2014. 68(1): p. 57.

[14] Tomlinson, M., et al., Scaling up mHealth: where is the evidence? PLoS medicine, 2013. 10(2): p. e1001382.

[15] Castelnuovo, G., et al., Chronic care management of globesity: promoting healthier lifestyles in traditional and mHealth based settings. Frontiers in psychology, 2015. 6: p. 1557.

[16] Mattila, E., et al., Personal health technologies in employee health promotion: usage activity, usefulness, and health-related outcomes in a 1-year randomized controlled trial. JMIR mHealth and uHealth, 2013. 1(2): p. e16.

[17] Krebs, P. and D.T. Duncan, Health app use among US mobile phone owners: a national survey. JMIR mHealth and uHealth, 2015. 3(4): p. e101.

[18] Kayyali, R., et al., Awareness and use of mHealth apps: a study from England. Pharmacy, 2017. 5(2): p. 33.

[19] Gibson, J.J., The theory of affordances. Perceiving, Acting and Knowing. Perceiving. Hillsdale, NJ: Lawrence Erlbaum Associates, 1977.

[20] Volkoff, O. and D.M. Strong, Affordance theory and how to use it in IS research. The Routledge companion to management information systems. New York: Routledge, 2018.

[21] Boudreau, M.-C. and D. Robey, Enacting Integrated Information Technology: A Human Agency Perspective. ORGANIZATION SCIENCE, 2005. 16(1): p. 3-18.

[22] DeSanctis, G. and M.S. Poole, Capturing the complexity in advanced technology use: Adaptive structuration theory. Organization science, 1994. 5(2): p. 121-147.

[23] Schultze, U. and R.J. Boland Jr, Knowledge management technology and the reproduction of knowledge work practices. The Journal of Strategic Information Systems, 2000. 9(2-3): p. 193-212.

[24] Markus, M.L. and M.S. Silver, A foundation for the study of IT effects: A new look at DeSanctis and Poole's concepts of structural features and spirit. Journal of the Association for Information systems, 2008. 9(10): p. 5.

[25] Leonardi, P.M., When does technology use enable network change in organizations? A comparative study of feature use and shared affordances. MIS quarterly, 2013: p. 749-775.

[26] Oyebode, O., et al. Persuasive Mobile Apps for Health and Wellness: A Comparative Systematic Review. in International Conference on Persuasive Technology. 2020. Springer.

[27] Liew, M.S., et al., Usability challenges for health and wellness mobile apps: mixed-methods study among mhealth experts and consumers. JMIR mHealth and uHealth, 2019. 7(1): p. e12160.

[28] Robles, N., et al., Evaluation Criteria for Weight Management Apps: Validation Using a Modified Delphi Process. JMIR mHealth and uHealth, 2020. 8(7): p. e16899.

[29] El Khoury, C.F., et al., A Dietary Mobile App for Patients Undergoing Hemodialysis: Prospective Pilot 
Study to Improve Dietary Intakes. Journal of Medical Internet Research, 2020. 22(7): p. e17817.

[30] Lau, N., et al., Android and iPhone Mobile Apps for Psychosocial Wellness and Stress Management: Systematic Search in App Stores and Literature Review. JMIR mHealth and uHealth, 2020. 8(5): p. e17798.

[31] Kanthawala, S., et al., Folk theorizing the quality and credibility of health apps. Mobile Media \& Communication, 2019. 7(2): p. 175-194.

[32] Leonardi, P.M., When flexible routines meet flexible technologies: Affordance, constraint, and the imbrication of human and material agencies. MIS quarterly, 2011. 35(1): p. 147-167.

[33] Strong, D.M., et al., A theory of organization-EHR affordance actualization. Journal of the Association for Information Systems, 2014. 15(2): p. 2.

[34] Balci, B., C. Rosenkranz, and S. Schuhen, Identification of different affordances of information technology systems: An empirical study. 2014.

[35] Choy, K. and D. Schlagwein. IT Affordances and Donor Motivations in Charitable Crowdfunding: The" Earthship Kapita" Case. in ECIS. 2015.

[36] Williams, C.K. and D.E. Wynn Jr, A critical realist script for creative theorising in information systems. European Journal of Information Systems, 2018. 27(3): p. 315-325.

[37] Lindberg, A., et al., Exploring configurations of affordances: The case of software development. 2014.

[38] Du, W.D., et al., Affordances, experimentation and actualization of FinTech: A blockchain implementation study. The Journal of Strategic Information Systems, 2019. 28(1): p. 50-65.

[39] Benbunan-Fich, R., An affordance lens for wearable information systems. European Journal of Information Systems, 2018: p. 1-16.

[40] Karahanna, E., et al., The Needs-Affordances-Features Perspective for the Use of Social Media. Management Review, 2018. 26(2): p. 298-310.

[41] Vishwanath, A., Getting phished on social media. Decision Support Systems, 2017. 103: p. 70-81.

[42] Wolff-Piggott, B. and U. Rivett, An activity theory approach to affordance actualisation in mHealth: The case of MomConnect. 2016.

[43] Struik, L.L., et al., The crush the crave quit smoking app and young adult smokers: qualitative case study of affordances. JMIR mHealth and uHealth, 2018. 6(6): p. e134.

[44] Dantu, R., R. Mahapatra, and J. Wang. Influence of Technology Affordance on the Adoption of Mobile Technologies for Diabetes Self-management. in International Conference on Smart Health. 2017. Springer.

[45] James, T.L., J.K. Deane, and L. Wallace, An application of goal content theory to examine how desired exercise outcomes impact fitness technology feature set selection. Information Systems Journal.

[46] Wong, C.C.K. and R.C.-W. Kwok. The effect of Gamified mHealth App on Exercise Motivation and Physical Activity. in PACIS. 2016.

[47] James, T.L., J.K. Deane, and L. Wallace, An application of goal content theory to examine how desired exercise outcomes impact fitness technology feature set selection. Information Systems Journal, 2019. 29(5): p. 1010-1039.

[48] Johnston, J.D., A.P. Massey, and C.A. DeVaneaux, Innovation in weight loss programs: a 3-dimensional virtual-world approach. Journal of medical Internet research, 2012. 14(5): p. e120.

[49] Volkoff, O. and D.M. Strong, Affordance theory and how to use it in IS research, in The Routledge Companion to Management Information Systems. 2017, Routledge. p. 232-245.

[50] Charmaz, K. and L.L. Belgrave, Grounded theory. The Blackwell encyclopedia of sociology, 2007.

[51] Oinas-Kukkonen, H. and M. Harjumaa, Persuasive systems design: key issues, process model and system features, in Routledge Handbook of Policy Design. 2018, Routledge. p. 105-123.

[52] Garcia, S.M., A. Tor, and T.M. Schiff, The psychology of competition: A social comparison perspective. Perspectives on Psychological Science, 2013. 8(6): p. 634-650.

[53] Bowman, N.D., D. Schultheiss, and C. Schumann, "I'm attached, and I'm a good guy/gal!": How character attachment influences pro-and anti-social motivations to play massively multiplayer online role-playing games. Cyberpsychology, Behavior, and Social Networking, 2012. 15(3): p. 169-174.

[54] Watts, M., Avatar self-identification, self-esteem, and perceived social capital in the real world: A study of World of Warcraft players and their avatars. 2016.

[55] Gardner, B., P. Lally, and J. Wardle, Making health habitual: the psychology of 'habit-formation'and general practice. Br J Gen Pract, 2012. 62(605): p. 664666.

[56] Johnson, S.C., et al., Neural correlates of selfreflection. Brain, 2002. 125(8): p. 1808-1814.

[57] Bawden, D. and L. Robinson, The dark side of information: overload, anxiety and other paradoxes and pathologies. Journal of information science, 2009. 35(2): p. 180-191.

[58] Broderick, J., et al., Designing health literate mobile apps. 2014: Institute of Medicine of the National Academies Washington, DC.

[59] Varshney, U., A model for improving quality of decisions in mobile health. Decision Support Systems, 2014. 62: p. 66-77.

[60] Liu, Y., Engineering aesthetics and aesthetic ergonomics: theoretical foundations and a dualprocess research methodology. Ergonomics, 2003. 46(13-14): p. 1273-1292.

[61] Beaudry, A., et al., Impact of IT User Behavior: Observations through a New Lens. Communications of the Association for Information Systems, 2020. 46(1): p. 15. 